\title{
AFECTIVIDAD, JUSTICIA Y POLÍTICA EN EL GORGIAS DE PLATÓN
}

\author{
Gustavo Gómez Pérez* \\ doi:10.11144/Javeriana.uph33-67.ajpg
}

\begin{abstract}
RESUMEN
Desde una lectura del diálogo Gorgias de Platón, la tesis del presente artículo es que, según la aproximación socrática a la justicia, el arte político propende por la asunción de la vulnerabilidad como apertura a la alteridad y al ejercicio de autonomía. La argumentación consta de tres partes. En primer lugar, se muestra que el reconocimiento de la vulnerabilidad conduce al reconocimiento del otro y, consiguientemente, a la valoración positiva de sus necesidades. En segundo lugar, se plantea que la justicia supone la autonomía, pues expresa el deseo de un bien común como ley autoimpuesta. La tercera parte expone cómo el conocimiento de lo justo se deriva de un trabajo dialéctico con el lenguaje, enfatizando su performatividad y su capacidad para movilizar emociones y deseos. Se concluye así que el saber político surge como un rapto hacia los límites de nuestras aspiraciones y posibilidades.

Palabras clave: Platón; justicia; política; afectividad; vulnerabilidad
\end{abstract}

* Pontificia Universidad Javeriana, Bogotá, Colombia.

Correo ealectrónico: gustavo-gomez@javeriana.edu.co

Para citar este artículo: Gómez Pérez, G. (2016). Afectividad, justicia y política en el Gorgias de Platón. Universitas Philosophica, 33(67), pp. 103-130. ISSN 0120-5323, ISSN en línea: 2346-2426, doi:10.11144/Javeriana.uph33-67.ajpg 


\title{
AFFECTIVITY, JUSTICE AND POLITICS IN PLATO'S GORGIAS
}

\author{
Gustavo Gómez Pérez
}

\begin{abstract}
Based on Plato's Gorgias, the thesis of this paper is that the Socratic approach to politics encourages the acceptance of our own vulnerability as a form of openness to otherness and as a way of enacting autonomy. The argumentation consists of three parts. First, it is shown that recognition of vulnerability leads to the acknowledgment of the other and, subsequently, to the positive valuing of the other's needs. Second, it is argued that justice presupposes autonomy, for it reveals the desire for the common good as a self-imposed law. Finally, the article shows how the knowing of what is just emerges from a dialectical labor with language, emphasizing its performativity and its capacity to provoke emotions and desires. It is concluded that the political wisdom springs up as a rapture towards the limits of our aspirations and possibilities.
\end{abstract}

Keywords: Plato; justice; politics; affectivity; vulnerability 


\section{Introducción}

EN el PRESENTE ARTículo SE USARÁ el téRMino “AFECTIVIDAD” para referir, de manera general, a la capacidad humana de ser afectado, vulnerado, transformado o movido por los comportamientos de los otros, lo que sugiere también nociones afines como la de emoción o pasión. En otras palabras, asumimos que el ámbito de la afectividad es la expresión esencial de la finitud y la fragilidad humanas, es la manera como se manifiesta en nosotros la conciencia de que estamos, en un sentido fundamental, a merced de los otros, lo que nos hace irremediablemente vulnerables. De acuerdo con esto, la afectividad se revela como un medium básico en el que se dan nuestras relaciones sociales. A partir de este planteamiento se podría afirmar que, en cierto sentido, los tejidos sociales se constituyen como tejidos afectivos.

Ahora bien, a pesar de ser expresión de nuestra comunión o vínculo esencial con los otros, la afectividad, el conjunto de nuestras disposiciones afectivas, no solamente opera como el soporte de vínculos sociales, sino también como la fuente de discordia y ruptura del tejido social. Es decir, la afectividad, en tanto expresión de nuestra finitud, revela el carácter esencialmente precario y singular de nuestra existencia y, así, motiva anhelos de poder e inclinaciones egoístas cuyo despliegue puede llevar, eventualmente, al interés de dominio irrestricto sobre otros. En otras palabras, justamente en tanto que la afectividad implica vulnerabilidad, nos hace anhelar estados de omnipotencia e independencia, esto es, genera el deseo de invulnerabilidad. Así pues, la afectividad, entendida como vulnerabilidad o capacidad para ser afectado, instiga tanto la constitución de un vínculo con otros como la división interna, la estratificación social de acuerdo con niveles de dependencia e independencia, y la vulnerabilidad e invulnerabilidad. De este modo, la relación entre afectividad y política se presenta en esencia como ambivalente.

En este orden de ideas, y considerando principalmente los planteamientos formulados en el Gorgias de Platón ${ }^{1}$, en el presente artículo se argumenta que el

1 Las referencias al diálogo Gorgias tendrán en cuenta tanto la edición en griego de Oxford University Press, con comentarios en inglés, de Dodds (1959), como la edición en castellano de Editorial Universitaria de Buenos Aires (Cappelletti, 1967). 
arte político se propone un adecuado ordenamiento o ajuste de la relación, siempre ambivalente, entre afectividad y política ${ }^{2}$. Específicamente, la tesis que defendemos es que la aproximación socrática a la justicia y el arte político se basa en un reconocimiento de nuestra vulnerabilidad y dependencia respecto de los otros como punto de partida para la búsqueda, desde el ejercicio pleno de la autonomía, de un bien común o universal. Esta tesis se desarrolla considerando tres ejes argumentativos. Por una parte, se muestra que la asunción de la propia vulnerabilidad favorece nuestra capacidad para ser transformados por los otros y, por ende, la consideración y aceptación de sus intereses y perspectivas. En relación con este punto se argumenta que nuestras disposiciones afectivas, en tanto expresiones de nuestra vulnerabilidad, hacen patentes los vínculos que nos ligan a los otros y, de este modo, nos obligan a asumir libre o autónomamente los intereses o puntos de vista de los demás. Esta línea argumentativa se articula con el planteamiento, sugerido por Sócrates en el mito que se narra al final del Gorgias, según el cual el mejoramiento propio y la aproximación al ideal del bien requiere del sufrimiento (Gorgias 525a-b). En la segunda parte del artículo se planteará que la justicia o el bien político se define en tanto que expresa un deseo o un ideal por lo común o lo universal, de manera que la verdadera política que profesa Sócrates jamás podría aspirar al sacrificio - por lo menos no el sacrificio involuntariodel individuo en función de un bien mayor. Dicho en términos fenomenológicos, el bien es, estructuralmente, un objeto de deseo, por lo que no puede ser, en sentido estricto, el bien sin ser deseado como tal. De acuerdo con esto, la última parte del artículo está dedicada a mostrar que el conocimiento de lo justo, el tipo de conocimiento propio de la política, se revela como el conocimiento derivado de una experiencia dialéctica del lenguaje, experiencia que supone una profunda conciencia de la dimensión performativa del mismo, y de su capacidad para movilizar emociones como, por ejemplo, la vergüenza. De acuerdo con esto, el bien común, el bien que, según lo plantea Sócrates, debe ser compartido

2 Teniendo en cuenta las indicaciones de Cappelletti sobre lo que significa el término $\tau \dot{\varepsilon} \chi \nu \eta$ en la Grecia antigua, entendemos aquí el término "arte", en sentido general, "abarcando toda actividad que suponga una experiencia anterior y se rija según ciertas reglas fundadas en ella" (Cappelletti, 1967, p. 257, n.6). De acuerdo con esto, usaremos indistintamente los términos "arte", "profesión" y "técnica". 
equitativamente por una comunidad ${ }^{3}$, es el bien que se revela como una ley que se asume de manera autónoma y como la expresión de los deseos más profundos del individuo. Así pues, el lazo más íntimo que une a una comunidad se revela como la experiencia de una cierta modalidad de la afectividad, de la vulnerabilidad, de la necesidad y del deseo del bien y la justicia.

\section{Superando intereses egoístas: la búsqueda del mayor bien y la necesidad del sufrimiento}

La postura que en el Gorglas asumen los personajes Polo y Calicles sobre la justicia, la libertad y el poder político, supone que las acciones humanas están, en su sentido más limitado, orientadas a la satisfacción de necesidades egoístas, propósito que se realiza dominando a los otros - en efecto, incluso Gorgias en una de sus intervenciones iniciales, sugiere que la libertad consiste en la capacidad de dominar a los otros (Gorgias 452d). Dicho dominio se basa en la manipulación de la fragilidad humana, en la capacidad para inspirar temor a la esclavitud, a la muerte, al exilio, al deshonor o al sufrimiento (Gorgias 466a-c, 483d-e). La capacidad para dominar o herir a los otros es considerada, en este caso, un gran poder y la manifestación paradigmática de la libertad humana ${ }^{4}$. Si tal poder se ejerce desde una situación de presunta invulnerabilidad y omnipotencia se hablaría de una situación en la que el tirano llega al poder total del Estado haciendo daño a los demás y evitando ser castigado por ello (Gorgias 470c-471d). Sócrates, como es de esperarse, rechaza esta perspectiva, cuestionando principalmente dos supuestos. Por una parte, está la creencia en que es posible una situación de poder absoluto sobre los otros y, por ende, un estado de invulnerabilidad frente a los demás. Por otra parte, se encuentra el supuesto según el cual es deseable someter a los otros buscando la satisfacción de inclinacio-

3 Sócrates muestra, de manera que parece aprobatoria, que la multitud considera que lo justo es preservar la igualdad (Gorgias 488e).

4 En efecto, como se sugiere más arriba, según la definición que da el mismo Gorgias al inicio del diálogo, la libertad se entendería comúnmente como la capacidad de dominio sobre los otros y, en últimas, lo que promete a sus potenciales discípulos es la posibilidad de poder y explotación de los otros. En palabras de Andrew Stauffer (2006, p. 27): "Promising them power and success, he [Gorgias]expresses no qualms at the prospect of manipulation and exploitation". 
nes corporales de carácter esencialmente egoísta. El problema, en este caso, es asumir que el bien derivado de satisfacer las inclinaciones corporales y los deseos egoístas es lo suficientemente significativo como para que valga la pena someter a los otros y hacerlos sufrir por su causa.

Para dilucidar las debilidades del primer supuesto examinaremos la reflexión socrática sobre las artes de la natación y la navegación, ya que ellas nos permiten evaluar la dimensión y alcance político del poder persuasivo de la retórica, que se presenta en la perspectiva de Gorgias - asumida luego por Polo y Calicles-, como el arte que nos permite dominar a los otros a través del discurso (Gorgias $452 \mathrm{~d}$-e). En este punto es importante subrayar que, desde la perspectiva de los interlocutores de Sócrates, la retórica no aparece simplemente como un medio de persuasión a través del discurso, sino como un arma política efectiva que permitiría condenar a muerte, despojar de bienes y desterrar a quien se desee (Gorgias 466b-e $)^{5}$; supuestamente, esta arma permitiría también proteger la propia vida y bienes.

Considerando esta comprensión del arte retórico como arma política, letal y efectiva, la refutación socrática pretende mostrar que ninguno de los supuestos poderes o capacidades que se le atribuyen a la retórica son exclusivos de la misma, pues la capacidad para persuadir, el uso del discurso, la capacidad para matar o herir arbitrariamente, así como el poder de evitar la muerte o proteger los bienes propios, pueden ser atribuidos también a otras artes o técnicas. En efecto, si el objetivo de la retórica fuese proteger la vida o los bienes, su poder no sería nada excepcional, haría sustancialmente lo mismo que la natación o el arte de la navegación, pues nadando protegemos nuestra vida evitando ahogarnos, y con el arte de la navegación se protegen la vida y bienes mientras se atraviesa el mar ${ }^{6}$. Así, pues, la refutación socrática se basa en los siguientes dos planteamientos básicos.

En primer lugar, Sócrates muestra que es absurdo evaluar el poder de un arte en función de su presunta capacidad para salvarnos o protegernos pues, en cierto

5 En este caso, según Polo, el tirano sería del modelo de un hombre feliz, capaz de satisfacer todos sus deseos y hacer siempre su voluntad, sin temer castigo o represalias por ello.

6 Aquí nos referimos a los ejemplos de la natación y la navegación que Sócrates examina en el Gorgias con el fin de mostrar que una vida buena no se limita al cultivo de prácticas que garanticen la supervivencia, en cierto sentido esto define un minimum y no un maximum de nuestras capacidades vitales. $\mathrm{Al}$ respecto, véase: Gorgias $511 \mathrm{e}-512 \mathrm{~d}$. 
sentido, todo arte responde a una cierta necesidad de supervivencia si bien, en últimas, el dominio de la vida y la muerte escapa a la capacidad de cualquier arte en cuanto tal. Específicamente, la comparación entre la retórica y el arte de la navegación hace patente que el hecho de presentar a la primera como un arma que sirve para hacer daño, para asesinar o despojar a alguien de sus bienes, supone una comprensión totalmente distorsionada de lo que debe ser un arte, de aquello que debe constituir esencialmente su poder. Por ello, sería sumamente absurdo que el piloto, en tanto que profesional de la navegación, se jactara de tener un gran poder por ser capaz de hacer morir, o hacer perder sus bienes, a quienquiera que viaje en su nave. Además, si se considera que la muerte es una condición esencial e ineludible del ser humano, algo obvio pero que la defensa que aquí se hace de la retórica parece pasar por alto, es claro que en últimas cualquiera puede dar la muerte a cualquier otro sin que para ello se requiera de ningún arte o conocimiento en especial; en este sentido, la capacidad para hacer morir a otros no se puede presentar como la virtud específica de un arte ${ }^{7}$.

En segundo lugar, y en contraste con el punto anterior, la refutación socrática evidencia que el poder de un arte se evalúa considerando su utilidad para la comunidad, esto es, su capacidad para producir un bien. En otras palabras, el poder político de un arte depende de qué tan grande o extenso es el bien que genera: el arte que produce un bien a muchos es más valioso, más poderoso políticamente, que el arte que solamente proporciona un bien individual, o a pocos. Inversamente, una acción o práctica que procure el bien individual a expensas del daño a la comunidad se revela como la antítesis del poder político, como expresión de impotencia. Asimismo, un arte que tenga un poder meramente reactivo, como por ejemplo la capacidad para evitar ciertos males, es menos poderoso que un arte que se define por la capacidad efectiva y positiva para producir bienes. En suma, si se supone que la retórica es un arte cuyo poder consiste en proteger la vida y los bienes, no sería en realidad un arte más poderoso que el de la natación y, definitivamente, sería menos poderoso que el arte de la

7 Esto es, precisamente, lo que trata de ilustrar Sócrates cuando sugiere que él también podría afirmar que tiene un gran poder si llegase al mercado con un puñal, con el cual podría quitar la vida, o despojar de sus bienes, a cualquiera de los allí presentes (Gorgias 469d-e). 
navegación, que por lo menos permite asegurar la pervivencia y la protección de bienes de varias personas, no solo de una.

De este modo se entiende por qué el diálogo cambia rápidamente el ejemplo de la natación por el del piloto de la nave, pues este en su práctica sería responsable de las vidas de otros, y no solamente de la suya propia. Con estos ejemplos se sugiere que, en cierto sentido, las artes sirven para paliar o resolver dificultades que surgen de nuestras carencias, pero esto no significa que su poder consista en manipular estas carencias en función de intereses propios. En este contexto teórico surge la compresión socrática de la justicia como el arte de mayor poder, pues si la justicia busca una distribución equitativa del bien en la polis, manteniendo así equilibradas todas las relaciones de la comunidad, entonces sus beneficios se presentan como de mayor alcance que los beneficios que procura el médico o, si fuese efectivamente un arte, la retórica. En efecto, si las artes o profesiones responden de una manera u otra a una vulnerabilidad o carencia humana, entonces esto significa que en últimas nuestra vida y bienestar en general depende de una adecuada orientación de las mismas. Mantener esta orientación sería el objetivo de la política. Más aún, el curso de la discusión con Polo y Calicles evidencia que en cualquier caso es necesario procurar el bien de todos los ciudadanos, y su educación en un adecuado sentido de la justicia, ya que al final de cuentas nuestra vida y bienestar puede depender de cualquiera de ellos, incluso sin que medie la práctica de un arte como tal. Esto se debe a que, como se indicó anteriormente, cualquiera puede hacer daño a cualquier otro sin necesidad de que cuente con un saber cualificado para ello; entonces, para la convivencia es necesario cultivar en los ciudadanos una buena voluntad y un sentido de justicia. En este punto vale la pena resaltar, aunque solo sea de manera preliminar, que una distribución justa o equitativa del bien implica, idealmente, un ordenamiento afectivo basado en la amistad o la buena voluntad entre los ciudadanos; de esta manera, no habría por qué asumir una actitud defensiva ante los otros, que es lo que hace el retórico.

Ahora pasemos a examinar el segundo supuesto criticado por Sócrates, aquel según el cual es deseable someter a los otros buscando la satisfacción de necesidades e intereses egoístas. Específicamente, nos concentraremos en un análisis del modelo, asumido por Polo, de Arquelao, el tirano supuestamente feliz que gobierna la polis y que perjudica a los demás sin ser castigado por ello. En este caso, el desafío de Sócrates consiste en demostrar que siempre es mejor 
hacer el bien — considerando la perspectiva universal, ideal, de la justicia - que hacer lo que parece bueno desde la perspectiva de intereses o inclinaciones egoístas $^{8}$. La defensa de esta tesis es un desafío para Sócrates justamente porque sus interlocutores parecen dispuestos, exclusivamente, a aceptar argumentos pragmáticos, argumentos basados en la posibilidad de una utilidad inmediata, para el individuo, de hacer el bien.

Enfrentando este desafío, Sócrates hablará de la salud del alma, que sería lo que produce la justicia, punto a ser examinado con más detalle en la segunda parte de este artículo. Por ahora nos interesa mostrar que los planteamientos socráticos tienen también un trasfondo pragmático que se basa en la relación entre arte o técnica y poder, pues según Sócrates lo que significa tener un alma saludable es, en cierto sentido, tener un alma capaz de discernir lo que es bueno para ella y la mejor manera de obtenerlo. En suma, el estado de salud del alma se define en términos de un poder o capacidad: el poder de lograr discernir y obtener el bien, un bien real y no meramente aparente.

Como se ha sugerido anteriormente, el poder de un arte depende de la magnitud o extensión de su injerencia: un arte es más poderoso dependiendo de su mayor rango de influencia social. Este planteamiento sobre el poder del arte sería aplicable también a la comprensión de la salud del alma: el grado de salud del alma depende de la magnitud de los bienes que ella puede discernir y alcanzar. Así pues, un alma que solamente es capaz de estimar como bienes los relativos a deseos y necesidades del propio cuerpo o la propia persona es impotente en comparación con un alma capaz de valorar como bienes los deseos y necesidades de una comunidad.

Entonces, suponiendo junto con Platón que ideales morales como el bien y la justicia definen el horizonte último del conocimiento y posibilidades humanos ${ }^{9}$, y considerando que estos ideales exceden los límites de lo dado empíricamente, es claro que no hay situación o hecho empírico capaz de satisfacer plenamente las

8 En su discusión con Polo, Sócrates distingue entre hacer lo que a uno le parece y hacer el bien verdaderamente (Gorgias 469e-470b).

9 Al respecto, cabe recordar que en la República (509b) la investigación sobre la justicia remite necesariamente a la indagación sobre la idea del bien, y se dirá que el Bien, con mayúsculas que enfatizan su carácter superlativo, se eleva más allá de la esencia, siendo no solamente principio de inteligibilidad de las cosas, sino también principio de su ser y desarrollo. 
aspiraciones humanas a la justicia y el bien ${ }^{10}$. En otras palabras, nuestros deseos últimos, aquello a lo que aspiramos con todas las capacidades de nuestra razón y conocimiento, necesariamente exceden los límites de lo que se da empíricamente. Esto significa que el bien y la justicia que anhelamos de manera definitiva y radical suponen la referencia a una comunidad ideal, universal, que incluiría a todos los otros. $\mathrm{Al}$ respecto, es importante recordar que en el Banquete se concibe el deseo como intrínsecamente ligado a una carencia o límite: se desea lo que no se tiene, o no se puede tener ${ }^{11}$. De este modo, la dinámica del deseo nos remite en últimas al plano de lo que carecemos de manera esencial en tanto que seres humanos, históricos, encarnados, a saber, lo ideal, lo imposible, lo divino o lo eterno. En consonancia con estos planteamientos sobre el deseo, se podría conjeturar que Sócrates concluye el diálogo con Calicles con un mito acerca de la muerte asumiendo que no podemos satisfacer nuestra aspiración a la justicia desde la perspectiva limitada por el cuerpo, nuestra vida histórica o nuestras circunstancias empíricas. En este sentido, la búsqueda de la justicia, que define el estado de salud del alma, supone la capacidad para asumir o aceptar la muerte o, de manera más general, los límites de nuestra existencia, como límites que no coinciden, por su estrechez, con los límites de nuestros deseos o aspiraciones. Se abre así la posibilidad de pensar, desear, actuar, en función de necesidades o posibilidades que exceden los límites y carencias que se imponen en nuestra vida material e histórica, necesidades o posibilidades que se proyectan hacia lo universal y absoluto. La justicia, en cuanto ideal, exige, entonces, la posibilidad de pensar o desear lo imposible, lo absoluto, la alteridad radical, lo que el ser humano no puede lograr u obtener por sí mismo: el alma justa se reconoce, pues, como carente de justicia y, por tanto, desea la justicia que no se ha dado, o que no se da históricamente, que no puede hacerse presente. De esta forma, por contraste, siempre tiene frente a sí una cierta conciencia

10 En el diálogo el Fedón se presentan argumentos que refuerzan este punto. Dice Sócrates: "si nuestra alma es inmortal, necesita de atención no sólo respecto a este tiempo cuya duración llamamos vivir, sino respecto a todo el tiempo, y el peligro ahora sí que parecería ser tremendo, si alguno se despreocupara de ella" (Fedón 107c).

11 La reacción estructural entre deseo y carencia es claramente ejemplificada en el Banquete, particularmente en el mito de Diótima sobre la concepción de Eros como hijo de Penía y Poros (Banquete 203b-204d). 
de la muerte, de su precariedad y finitud, de los límites estrechos que definen sus perspectivas e intereses particulares.

Siguiendo esta línea argumentativa, el tirano no solamente aparece como un ser impotente en tanto que sus acciones no aportan ningún beneficio — según lo muestra Sócrates, ni siquiera aportan beneficios al agente, al tirano-, sino también como un alma que se puede caracterizar como enferma y mutilada, marcada por la privación. La privación consiste, en este caso, en que, encapsulada en su perspectiva meramente particular, el alma del tirano se vuelve incapaz de pensar en el otro, y de integrarse así a la vida de una comunidad: esta persona está, pues, desmembrada, desvinculada socialmente, confinada a la existencia más mínima. De esta manera, el alma del tirano se revela como infértil e incapaz de ir más allá de sí misma; no podría, en realidad, procrear, desear la existencia de un otro, proyectar un terreno común de encuentro con el otro, lo que significa que su relación con el otro siempre estará marcada por la enemistad o la discordia, la heteronomía. Así, el otro, del que siempre, inevitablemente, se depende, es visto como negación de sí. Evocando, nuevamente, planteamientos centrales del Banquete, se podría decir que en principio el tirano, en su caracterización más radical - que es justamente aquella que se da en el Gorgias -, no puede engendrar desde sí ninguna referencia a lo común o universal; así, contrariamente a lo que serían, quizás, las aspiraciones más íntimas de su orgulloso carácter, el tirano se aleja de toda forma de participación de la inmortalidad ${ }^{12}$.

$\mathrm{Al}$ renunciar a toda forma de participación de la inmortalidad, el tirano se termina alejando de la tan anhelada invulnerabilidad. Efectivamente, en tanto que finito el ser humano solamente puede participar de la inmortalidad sobrepasando sus límites, exponiéndose a la alteridad de los otros y, eminentemente, en la alteridad radical de lo ideal, cuya encarnación más concreta se da en el ámbito de lo político: lo común, lo universal. Esta exposición a la alteridad supone, en cierto sentido, abandonarse a sí mismo y lo que se posee, ir más allá de sí, es decir, experimentar diversas modalidades de la muerte, asumir la muerte. De este modo, la exposición a la alteridad y, particularmente, a la alteridad radical de lo

12 En su falta de generosidad y compasión, el tirano injusto no podría ser ni un verdadero padre ni un verdadero gobernador, pues ambas posibilidades suponen la priorización de intereses diferentes a los propios, es decir, la asunción de una legalidad que se impone desde un otro. 
ideal, supone el cuestionamiento constante de los límites que definen al sí mismo, los límites de la propia existencia: la existencia plena se comprende, entonces, como fundamentalmente extática, fuera de sí.

Por su parte, la retórica, en tanto que arma que permite tomar el poder político y mantenerlo dominando o sometiendo a los otros, procura mantenerse fuera del alcance del otro, de su ley. La retórica busca proteger los bienes, la vida, la reputación y el orgullo, disfrazando o maquillando las propias creencias y acciones para persuadir en los tribunales y evitar castigos, todo esto fuera de la ley del bien común. En consonancia con esta función de la retórica, el modelo del tirano feliz sería aquel que hace lo que le parece durante todo su gobierno, impunemente, es decir, sin la asunción de ningún tipo de legalidad. Así, pues, la retórica se presenta como un medio para convertirse en alguien solo aparentemente invulnerable. En efecto, al negar la referencia a toda ley el tirano se entrega a la heteronomía absoluta o padecimiento más fatal: el caos, la necesidad que se impone sin principio, sin ley, sin regularidad, caprichosamente; el tirano es, así, en sí mismo esclavo de la tiranía. De esta manera, abrazando el caos de lo efímero, el tirano renuncia a su libertad, a su autonomía, a la posibilidad de gobernarse a sí mismo. En relación con este punto, cabe destacar que una característica de los personajes Polo y Calicles es que parecen estar caprichosamente sometidos al vaivén de opiniones y creencias que no han sido apropiadas, cuyas implicaciones se desconocen y que, eventualmente, se presentan para ellos mismos como indeseables, como ajenas. Incapaces de asumir la obediencia a la ley, que es siempre de alguna manera ley del otro, Polo y Calicles no parecen tolerar los acuerdos comunes que impone la refutación socrática ${ }^{13}$. Así, ellos son incapaces de un gesto genuino de amistad, pues la amistad supone la valoración de intereses ajenos como íntimamente ligados a los propios, y estos personajes se niegan a aceptar, por lo menos teóricamente, un modo de pensar que abra esta posibilidad. De acuerdo con esto, se podría decir que la invulnerabilidad que busca el orador, así como la que

13 Aunque con frecuencia el tono parece irónico, en diferentes puntos del diálogo Sócrates insiste en la amistad, en el interés por la salud del otro, por sacar al otro del error, como propósito del ejercicio de la dialéctica. En concreto, en una parte central del diálogo con Calicles, Sócrates afirma explícitamente que las tres condiciones para examinar el alma son las siguientes: "ciencia, buena voluntad, y franqueza” (Gorgias 487a), lo que significa que la amistad, como expresión fundamental de buena voluntad hacia el otro, es condición para el arte de la justicia. 
pretende el tirano, supone una especie de muerte en vida: la existencia del político retórico no estaría abierta al otro, a una comunidad, está siempre en relación de enemistad o discordia con el otro; así, está siempre en riesgo, patentemente al borde de la muerte ${ }^{14}$.

En contraste con las pretensiones de la retórica, en el Gorgias, la dialéctica se presenta como una técnica que pone en juego la vulnerabilidad humana, particularmente, apelando a la vergüenza e, indirectamente, al orgullo, como emoción fundamental ${ }^{15}$, ya que ella pretende llegar al núcleo de la ley que ha sido interiorizada desnudando los afectos y opiniones que la enmascaran y propendiendo así por una apropiación y explicitación de las propias leyes y principios de acción; de este modo, la dialéctica puede ser entendida como una especie de entrenamiento en el gobierno. La dialéctica, en efecto, nos enseñaría a revelar la alteridad que ha sido internalizada, asumida, aquello que ya, desde siempre, nos ha afectado o vulnerado; es más, podríamos decir que la alteridad interiorizada nos ha doblegado, ya que es como una ley que el otro ha impuesto y que uno ha terminado por asumirla libremente, esto es, como ley propia, ley que se dicta a sí mismo. En este sentido, la vergüenza sería, en efecto, manifestación de la aceptación de la ley que el otro o los otros han impuesto ${ }^{16}$. De este modo, se revela un aspecto fundamental de los alcances políticos de la dialéctica pues, según lo planteado anteriormente,

14 Efectivamente, Sócrates afirma, con base en una cita de Eurípides, que "quizás nosotros en realidad estamos muertos" (Gorgias 492e-493a), sugiriendo que quizás lo que llamamos usualmente vida, que es lo que tanto parece valorar Calicles, no es otra cosa que una forma de muerte.

15 Sobre el uso dialéctico de la vergüenza vale la pena considerar, como ejemplo, la manera como la

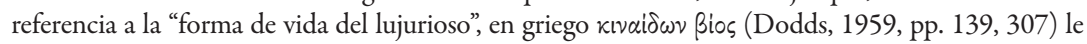
sirve a Sócrates para conmocionar a Calicles y llevarlo a admitir la distinción entre placeres buenos y placeres malos. En relación con este punto, y para entender el poder retórico de la intervención socrática, es importante tener en cuenta que el término kıvai $\omega \omega \nu$ alude al que es pasivo en la relación sexual homosexual (Nussbaum, 1995, p. 204), lo cual puede ser muy insultante para alguien como Calicles que inicia su intervención criticando la pasividad contraponiéndola a valores de hombría o virilidad (Gorgias 482c-486e). Sobre el tema de la vergüenza es importante anotar que la dimensión política de la vergüenza en el Gorgias es ampliamente examinada por Tarnopolsky (2010), quien sostiene lo siguiente: "I argue that Plato's analysis of shame in the Gorgias supplies a deeper understanding of the necessary but dangerous role of this emotion in human life and democratic politics than either the proponents or the opponents of the contemporary politics of shame and civility" (pp. 29-30).

16 En este punto es importante recordar que la vergüenza, en tanto enfatiza nuestra vulnerabilidad y nuestra dependencia respecto de la perspectiva y opiniones ajenas, puede tener efectos políticos tanto negativos como positivos; en este caso, estamos enfatizando lo que serían efectos positivos. 
para sintonizarse con un bien común es necesario que el individuo se libere y se abandone a sí mismo, asuma sus límites o incluso su muerte, como expresión de su propia libertad: la dialéctica sería el ejercicio mismo de esta libertad.

En suma, para concluir esta sección, afirmamos que, si bien, la vulnerabilidad es una condición esencial de lo que significa ser humano, frente a esta condición hay fundamentalmente dos alternativas, dos comportamientos posibles: o bien, uno se comporta pretendiendo ser invulnerable, como haría el tirano, y como sugiere de alguna manera la perspectiva sobre la retórica de Polo y Calicles; o bien, uno se comporta asumiendo la propia vulnerabilidad y finitud, el padecimiento o sufrimiento del otro o los otros, como lo sugiere la posición socrática y su uso de la dialéctica. En el primer caso, pretendiendo invulnerabilidad, se cae en el riesgo, paradójicamente, de sufrir un mal terrible: renunciar a toda posibilidad genuina de trascendencia y confinarse a una vida en los límites de su mínima expresión, a saber, la perspectiva estrictamente individual en la que se hace imposible la comunión con otros. Por el contrario, al asumir la vulnerabilidad, la posibilidad del sufrimiento y la muerte, la posición socrática se presenta como una manera de presionar o desafiar los límites que imponen los intereses y deseos egoístas, de manera que estos se acerquen a lo que sería un bien ideal, común, universal. Cabe enfatizar aquí que la capacidad para enfrentar la muerte y el sufrimiento se puede considerar, en sí misma, como expresión de una cierta posibilidad de trascendencia: la posibilidad, siempre abierta, de desafiar los límites que imponen en la existencia circunstancias históricas o empíricas. Desde este punto de vista, el sufrimiento humano se presenta como contracara de la libertad, puesto que esta se da paradigmáticamente en la superación de resistencias, límites, superación que implica sufrimiento. Sin embargo, de esta aproximación al sufrimiento no se debe concluir que, desde la perspectiva socrática, la búsqueda del bien común, universal, de la polis o el Estado, justifica el sufrimiento o el sacrificio del individuo. Para aclarar este punto será necesario dilucidar, de manera precisa, cómo para Sócrates se define el verdadero político, etiqueta que, por lo demás, él se atribuye a sí mismo (Gorgias 521d).

\section{El arte político y el cuidado del individuo}

Hasta AHORA HEMOS DEFENDIDO LA TESis según la cual la dialéctica y la retórica se oponen, fundamentalmente, en tanto que la una asume y la otra niega la 
vulnerabilidad. También hemos mostrado que la vulnerabilidad no es meramente manifestación de nuestras carencias y límites sino que es, asimismo, expresión de una capacidad de trascendencia. Esta capacidad de trascendencia parece ser, además, condición necesaria para la constitución de una comunidad política; esto en virtud de que hace patente la dependencia mutua de los miembros de una comunidad, incentivando así la búsqueda de un bien ideal, común y universal. De acuerdo con esto, una justa valoración del importe político de la vulnerabilidad humana presupone una clara comprensión de lo que podríamos denominar su dimensión positiva como condición que permite la cohesión social en función de un bien común, lo que se contrapone a lo que se consideraría como su dimensión negativa en cuanto condición que facilita la manipulación o el dominio de aquellos que están sometidos al poder de un individuo o grupo. En efecto, si se asume la vulnerabilidad y la finitud como condiciones esenciales de lo humano, es claro que todo poder político es esencialmente precario porque quienquiera que esté en el poder siempre podrá ser vulnerado por los otros; por lo tanto, solamente podrá estar en el poder en tanto que soportado o avalado por los otros. De acuerdo con esto, desde la perspectiva socrática el único verdadero poder deriva de la referencia al bien, el bien de la polis que es común, universal, esto es el bien que necesariamente debe ser compartido con los otros y que, en tanto ideal, es un bien siempre por venir, siempre elusivo en relación con cualquier intento de particularización.

Una vez reconocido el imperativo político del bien común, sin embargo, es necesario definir en qué medida, desde la perspectiva socrática, esto no supone la legitimación del sacrificio del individuo que es, paradójicamente, lo que el destino de Sócrates parece ilustrar. Según se intentará demostrar en lo que sigue, la posición socrática implica que no es deseable sacrificar al individuo a un bien mayor, a menos que dicho sacrificio sea aceptado o deseado por el individuo mismo. Dicho en otros términos, la realización del bien, incluso si se trata de un bien universal, requeriría del consentimiento individual. Para avanzar en la comprensión de este problema es importante distinguir entre lo que significa la vulnerabilidad como potencialidad para el sufrimiento o padecimiento, y la dimensión puramente actual del sufrimiento o padecimiento. En efecto, la vulnerabilidad o afectividad conlleva siempre una dimensión potencial, irreductible a mera actualidad, y supone una posibilidad de acción. De acuerdo con esto, se puede decir que la condición de vulnerabilidad no se reduce jamás a mera pasividad. Incluso en el 
padecimiento extremo, por ejemplo, en la tortura, está implícita la referencia a una potencialidad, a una dimensión no actual: la tortura, en efecto, no supone meramente la actualidad del dolor, sino la posibilidad de su prolongación o extinción. A la luz de esta distinción, se entrevé que la dimensión positiva, en relación con la política, de la vulnerabilidad depende de su ser potencial y no actual. La posibilidad siempre abierta de afección es lo que lleva a establecer pactos de mutuo acuerdo con los otros, incluso habría que considerar la posibilidad de una afección virtual, después de la muerte, en relación con el sufrimiento de amigos, de descendientes y, en últimas, de la humanidad en general a la cual se está íntimamente ligado. Así, según lo hemos indicado anteriormente, la dialéctica filosófica asumiría la tarea, no irrelevante para la política, de revelar la vulnerabilidad potencial que nos caracteriza, necesariamente, en tanto somos seres humanos y, por extensión, en tanto que somos ciudadanos.

$\mathrm{Al}$ considerar este énfasis en la potencialidad de la vulnerabilidad como condición de la política parece necesario matizar lecturas como la de Martha Nussbaum (1995, pp. 198-205), que resaltan el "racionalismo" platónico y que muestran que la perspectiva socrática sobre la virtud tiende a menospreciar aspectos concretos de lo que es el amor, el deseo o, en general, la dimensión sensual de la existencia. De acuerdo con esta lectura, Sócrates ignoraría aspectos concretos concernientes a la relación entre afectividad, deseo y política. Esto es también lo que Calicles sugiere si bien, por otra vía argumentativa, en su primer discurso dirigido a Sócrates (Gorgias 482c-486e), según el cual la filosofía hace del ciudadano un ser indefenso que es incapaz de intervenir efectivamente en la política, por lo que estaría sometido al dominio de los otros, con lo que se prueba, supuestamente, la incompatibilidad entre la filosofía y la política. El juicio de Sócrates, como fue sugerido anteriormente, parece confirmar la afirmación de Calicles, pues en él Sócrates se muestra, en cierto sentido, torpe e incapaz de salvar su vida. Paradójicamente, desafiando estas opiniones, en el Gorgias (521d), Sócrates afirma explícitamente ser el único en Atenas que practica el verdadero arte político; esta afirmación implicaría que la dialéctica es en sí misma un genuino trabajo político.

En relación con este punto, y teniendo en cuenta nuestros planteamientos previos sobre la vulnerabilidad, la pregunta que debemos considerar ahora se refiere, precisamente, a la exigencia que significa ser un verdadero político, además de examinar en qué sentido la política es un arte (una práctica acompañada de logos). 
En el Gorgias se sugiere que la verdadera política se puede identificar con lo que sería el arte de la justicia. De hecho, Sócrates trata indirectamente esta cuestión cuando define la retórica como un tipo de adulación que suplanta ilegítimamente a la justicia, así como la cocina suplanta a la medicina (Gorgias 464b-466a). La comparación entre la justicia y la medicina permite inferir que la justicia basada en un conocimiento verdadero de la naturaleza del alma procuraría su salud, mientras que la retórica pretende intervenir en el alma sin conocer realmente su naturaleza o disposición interna. La cuestión más específica que se debe examinar a la luz de estos planteamientos se refiere, entonces, al tipo de conocimiento que guía el arte de la justicia.

Según lo que se sugiere en el contexto de la analogía entre justicia y medicina, el arte de la justicia tiene que ver con un conocimiento sobre lo que es bueno o malo para el alma, así como el médico puede identificar lo que es bueno o malo para el cuerpo. Siguiendo esta equivalencia se podría decir que la justicia establece orden en el alma, de manera que esta alcance su excelencia, así como la medicina procura restablecer el orden en el cuerpo. Así, el problema nuclear consistiría en definir cuál es el paradigma o la medida según la cual se determina este adecuado ordenamiento del alma.

Como lo hemos sugerido anteriormente, la salud del alma depende de su articulación con un bien mayor; en consonancia con esta perspectiva ahora podríamos añadir que en el Gorgias (488e) Sócrates mismo, encarnando la voz de la multitud, define la justicia como distribución equitativa del bien, si bien esta acepción de la justicia surge de una refutación a Calicles y concierne a la distinción entre justicia natural y convencional (Gorgias 488c-489b). Según Calicles, la justicia convencional es derivada de la multitud de individuos débiles e incapaces de imponer sus propios intereses; la justicia natural, por el contrario, es la justicia que imponen individuos fuertes, que dominan a los otros para la satisfacción de sus propios intereses y deseos. Sócrates refuta esta posición mostrando en primer lugar que, la multitud de débiles, en tanto que multitud, es más fuerte que cualquier individuo fuerte, de manera que tendrían el derecho "natural" a imponer sus intereses. En segundo lugar, Sócrates afirma que esa misma multitud asume que la justicia consiste en una distribución equitativa del bien. De esta manera, retomando los planteamientos desarrollados en la primera parte del presente artículo, podemos decir que la ordenación o regulación del alma, su salud, depende de su 
adecuada participación en el bien común, de manera que los intereses individuales concuerden con el interés por una distribución equitativa del bien.

Esta conclusión, no obstante, parece implicar lo que se denominaría una perspectiva democrática que reivindica el valor de los intereses de la mayoría, sin que estos estén cualificados de una manera particular, lo que parece inconsistente respecto del desprecio que Sócrates manifiesta explícitamente, y con frecuencia en el Gorgias, hacia la multitud. Sin embargo, a continuación proponemos una clave de lectura según la cual la posición socrática no es necesariamente inconsistente. Para ello, en primera instancia, planteamos no equiparar la distribución equitativa del bien que refiere Sócrates a la definición de justicia a una mera distribución de bienes materiales.

En relación con este punto cabe resaltar que al considerar lo justo, Sócrates habla del bien o del mayor bien y no simplemente de bienes, lo que evoca un bien superlativo (Gorgias 502e, 503d-e, 513d) ${ }^{17}$. ¿Cómo interpretar, pues, este bien superlativo en términos políticos? Hemos visto que la justicia opera sobre el alma, desde lo que se infiere que el bien al que se refiere Sócrates como aquel al que aspira también la multitud tiene que ver con la salud del alma. Si bien, parece difícil asumir que Sócrates tiene en mente algo así como un alma de la multitud, la refutación a Calicles sugiere que la multitud puede actuar, ocasionalmente, como un individuo, como unidad, sobre todo cuando promulga leyes (Gorgias 488c). De este modo, parece que el bien político, la justicia, es la excelencia del alma -es decir, la máxima expresión de lo que es un principio vital, un principio del movimiento y de la vida-. Por su parte, la autonomía, entendida literalmente como ley de sí, como la legalidad en sí misma, se impone como principio absoluto, como alteridad y autoridad radical: la raíz de la ley. En otras palabras, el bien político solamente puede ser político, común, universal en tanto que superlativo, es decir: singularidad irremplazable y no negociable de la ley que se impone desde sí misma - esta ley es la autonomía misma que prevalece tanto en la multitud como en el individuo-. Incluso dentro de un mismo individuo, sugiere Sócrates, existe una referencia a la alteridad radical de la ley, a su singularidad inexpugnable, a la autonomía, lo que permite hablar de un gobierno de sí (Gorgias 491d). Ahora bien,

17 El término que usa Sócrates en estas ocasiones, cuando se habla del "mayor bien”, es $\beta \varepsilon ́ \lambda \tau \tau \sigma \tau o \nu$, que es un modo superlativo de àyatòs. 
¿cómo se conoce o reconoce esta autoridad de la ley? Puesto que la ley es en últimas ley de sí, autonomía, solamente es reconocible como singularidad: la ley justa es incomparable, inconmensurable, escapa a toda aplicación convencional, a toda parametrización vulgar. De acuerdo con esto, la ley de sí, solamente es reconocible como un polo afectivo, como un objeto de deseo, como el reconocimiento oscuro de una carencia o de una no presencia, como el rastro de la otredad. Por ello, uno mismo no puede explicar, en últimas, por qué quiere lo que quiere profundamente, o por qué teme lo que teme profundamente. Así, la experiencia afectiva se presenta como la experiencia de una alteridad radical. El sí mismo está, pues, siempre subordinado a un otro, autónomo, que no es un otro conocido o conocible, es un otro que se ama, se desea, se espera, se teme y se respeta. Esta interpretación del bien político superlativo, de la justicia, explicaría el interés que Sócrates asume como esencialmente político en el examen particular de las creencias íntimas del individuo, de los principios, siempre oscuros, que rigen la acción de un individuo y definen su autonomía.

Dicha posición socrática respecto de la política puede ilustrarse con base en una lectura del diálogo Eutifrón, en el que se trata el tema de la justicia en relación con la piedad. En este diálogo se narra que Eutifrón se dirige a acusar a su padre de cometer una injusticia, un asesinato injustificado, pasando por alto los mandatos de los dioses. Con evidente ironía Sócrates subraya que Eutifrón debe tener un conocimiento pleno de estas cuestiones si se atreve a acusar a su propio padre de injusticia e impiedad, situación que Eutifrón utiliza para resaltar su virtud. La posición de Eutifrón se resume en el siguiente pasaje del diálogo:

Eut-Es ridículo Sócrates, que pienses que hay alguna diferencia en que sea extraño o sea familiar el muerto, y que, por el contrario, no pienses que es solo necesario tener en cuenta si el que lo mató lo hizo justamente o no. Y si lo ha hecho justamente, dejar el asunto en paz; pero si no, perseguirlo, aunque el matador viva en el mismo hogar que tú y coma en la misma mesa. En efecto, la impureza es la misma si, sabiéndolo, vives con él y no te libras de ella tú mismo y lo libras a él acusándole en justicia. En este caso, el muerto era un jornalero mío. Como explotamos una tierra en Nasos, estaba allí a sueldo con nosotros. Habiéndose emborrachado e irritado con uno de nuestros criados, lo degolló. Así pues, mi padre mandó atarlo de pies y manos y echarlo a una fosa, y envió aquí a un hombre para informarse del exégeta sobre qué debía hacer. En este tiempo se despreocupó del hombre atado y se olvidó de él en la 
idea de que, como homicida no era cosa importante si moría. Es lo que sucedió. Por el hambre, el frío y las ataduras murió antes de que regresara el enviado al exégeta. A causa de esto, están irritados mi padre y los otros familiares porque yo, por este homicida, acusé a mi padre de homicidio; sin haberlo él matado, dicen ellos, y si incluso lo hubiera matado al ser el muerto un homicida, no había necesidad de preocuparse por un hombre así. Pues es impío que que un hijo lleve una acción judicial de homicidio contra su padre. Saben mal, Sócrates, cómo es lo divino acerca de lo pío y lo impío. (Eutifrón 4b-e)

En relación con nuestra reflexión sobre la política en el Gorgias, el pasaje citado llama la atención porque parece ejemplificar un caso paradigmático de devoción a la justicia, pues Eutifrón acusa a su padre defendiendo, supuestamente, las leyes establecidas y, además, los intereses de un extraño - persiste, no obstante, la duda respecto de si Eutifrón está interesado sobre todo en defender sus intereses particulares, pues el muerto era un jornalero suyo-. Aquí es importante recordar que en el Gorgias, en su diálogo con Polo, Sócrates sugiere, si bien, con cierta ironía, que la retórica sería útil para persuadir a nuestros amigos o parientes de acudir ante la justicia, a recibir el castigo correspondiente por sus faltas, cuando la situación así lo requiera (Gorgias $480 \mathrm{~b}-\mathrm{e}$ ). De acuerdo con esto, se podría pensar que la actitud de Eutifrón sería aquella que Sócrates alabaría en sus conciudadanos, un ejemplo de virtud intachable, en tanto que parece implicar una subordinación voluntaria de intereses propios a la universalidad de la ley. Sin embargo, la reacción de Sócrates ante Eutifrón es de perplejidad, no solamente por la confianza que manifiesta en su propio conocimiento sobre la justicia, sino también por su resoluta disposición a acusar a su padre. Esta sorprendente situación, añadida al hecho de que Sócrates viene a los tribunales a responder por acusaciones de impiedad, lleva a Sócrates a examinar las opiniones de Eutifrón en relación con la justicia y la piedad, indagación que el propio Eutifrón interrumpe abruptamente.

Como es de esperar, Sócrates hace patente que Eutifrón no tiene conocimiento cierto de lo que es la justicia, pues su presunto conocimiento sobre esta cuestión se limita a vagas fórmulas sin contenido concreto como: la justicia sería aquello que place a los dioses. Así, el diálogo termina aporéticamente sin alguna definición de lo que es la justicia. 
Ahora bien, aunque en este diálogo no proporciona una definición de la justicia es posible derivar de él algunas indicaciones importantes sobre el tema. Específicamente, el Eutifrón muestra cuán peligroso puede ser aceptar acríticamente una definición convencional de justicia asumida como referente inequívoco para juzgar acciones particulares. Cabe recordar que la acepción convencional de justicia asumida por Eutifrón pasa por alto la consideración del estado del alma de los individuos involucrados en la situación, de su singularidad. En este sentido, queda la sospecha de si el juicio de Eutifrón es injusto en tanto no considera cuidadosamente - y podríamos incluso decir, amorosamente - la singularidad del acusado, en este caso, su propio padre. De este modo, se llama la atención sobre una discordancia fundamental entre la regla o la ley convencional, impersonal y explícita, y las acciones particulares. En este caso, no es en absoluto evidente que el padre de Eutifrón sea un asesino según la ley proclamada para acusarlo como tal, y tampoco lo es que Eutifrón sea un verdadero paladín de la justicia. Esta discordancia se evidencia en el diálogo de múltiples maneras. En primer lugar, cuando se le pide explicar qué significa que una acción justa sea aquella que place a los dioses, Eutifrón se muestra incapaz de dar cuenta, adecuadamente, de esta definición y termina en contradicciones. De este modo, Sócrates revela la ignorancia de Eutifrón respecto de la justicia sugiriendo, además, que su acción, esto es, la acusación a su padre, puede ser injusta a pesar de concordar aparentemente —o precisamente porque concuerda - con una comprensión convencional de lo que es la ley jus$\operatorname{ta}^{18}$. Así, pues, parece que el error de Eutifrón consiste, por una parte, en emitir un juicio moral sin haber examinado cuidadosamente los presupuestos del mismo ${ }^{19}$, y por otra, en aplicar este juicio a un individuo sin considerar la posición particular del acusado en relación con la justicia. Idealmente, en lo que sería un juicio perfectamente justo, es de esperar que incluso el acusado mismo debe abrazar

18 Es importante considerar, sin embargo, que la posición "convencional" de Eutifrón es inconsistente, no solo en tanto que se muestra incapaz de dar cuenta del sentido de la norma, abriendo así, en la ignorancia, el espacio para la injusticia, sino que además viola otras convenciones que parecen tener un sentido que es menos equívoco, como la convención que nos obliga a cuidar de nuestros padres.

19 Queda la pregunta acerca de si, en últimas, todo juicio explicitado, todo juicio dado, establecido, formulado, respecto de un otro, quienquiera que este otro sea, es en sentido estricto injusto. 
como justa la ley que lo juzga ${ }^{20}$, pero Eutifrón no considera, por ejemplo, si en algún momento su padre pretendió cometer injusticia, asesinar, o si la muerte del esclavo no se podría interpretar como una fatalidad. Este ejemplo muestra que la correlación entre una ley universal y la dimensión moral de los actos particulares de un individuo no se puede evaluar con base, exclusivamente, en la dimensión exterior de los actos y los contenidos formales de la ley; antes bien, esta correlación entre ley y acción debe ser legitimada desde la autonomía del individuo que funge como agente. En este orden de ideas, las inconsistencias en el discurso de Eutifrón pueden ser vistas como ilustración de las dificultades que surgen, inevitablemente, cuando se trata de aplicar normas o convenciones generales para evaluar, de manera vertical e irreflexiva, la situación de un individuo en relación con la justicia. Según mostraremos a continuación, la justicia no es un arte basado en un conocimiento "objetivo" e impersonal, un arte en el cual los casos particulares encajan perfectamente en principios generales.

Llegados a este punto, vale la pena recordar que para Sócrates la justicia se define literalmente como un estado del alma, por lo que para entender cómo se da el gobierno del alma es necesario considerar, primero, que el alma es un tema de estudio muy particular, pues se trata de un "objeto" inmaterial, lo que significa que sus estados no son visibles o reconocibles como algo físico. En sentido estricto, el alma no puede ser asumida como un objeto porque nunca está presente. Por esta razón, puesto que no hay elementos visibles que se puedan asumir inequívocamente como indicios de la salud del alma, es necesario recurrir siempre a un método indirecto, a un examen del lenguaje y las opiniones, que se entreteje con el trasfondo de creencias y afectos que definen los principios de acción y las leyes que el individuo se da a sí mismo: su autonomía. Esto parece ser, según se muestra en el Gorgias, lo que supone Sócrates al afirmar que él es el único verdadero político de Atenas, punto que se podría esclarecer a partir de un examen de la relación entre el lenguaje y el arte de la justicia. Veamos.

20 De hecho, para que una pena sea justa es necesario que el acusado, de alguna manera, la reconozca como tal. Si el acusado no logra realmente ver la injusticia en su acción, entonces la ley no puede valer como tal para él. De aquí la importancia política del buen entendimiento de la persuasión y la retórica. 


\section{La dimensión performativa del lenguaje político}

Como fue Sugerido ANTERiormente, en el Gorgias, el tema de la justicia surge en relación con un examen de la retórica, e implica una referencia al lenguaje, pues la retórica se presenta inicialmente, en palabras del mismo Gorgias, como un tipo de discurso acerca de lo justo y lo injusto. En este orden de ideas, Sócrates precisa que la retórica produce discursos acerca de lo justo y lo injusto pero sin conocimiento, como forma de adulación que suplanta a la justicia, solamente apelando a lo agradable. De esta manera, teniendo en cuenta el paralelismo entre retórica y justicia que plantea Sócrates, se podría decir que la justicia es un arte de discursos que pretenden persuadir sobre lo justo y lo injusto, lo bueno y lo malo, con verdadero conocimiento ${ }^{21}$. Igualmente, es posible concluir que el verdadero conocimiento al que debe aspirar el político es sobre el estado del alma, esto es, sobre los principios que rigen, en últimas, la vida de un individuo, considerando la manera como la autoridad de estos principios se evidencia en el discurso. Desde esta conclusión se explica, además, por qué Sócrates insiste en llegar a una definición dialéctica de la justicia en el diálogo, pues aquello que importa no es simplemente una definición convencional e impersonal de esta virtud sino, más bien, revelar el estado del alma del individuo examinado, revelar qué es lo que alguien realmente quiere y piensa. De este modo, la dialéctica es lo que permite, de hecho, un reconocimiento del alma como tal, es decir, de aquello que mueve a un individuo, lo que dirige y rige sus acciones. Entonces, el alma justa sería aquella que reconoce plenamente sus propios principios de acción, afectos, deseos y aspiraciones más profundas.

Si retomamos el caso de Eutifrón, e igualmente la imagen de los oradores en el Gorgias, la importancia del rigor lógico y terminológico en el proceso de argumentación está, en cierto sentido, subordinada a la dimensión performativa del lenguaje $\mathrm{e}^{22}$. Lo que importa es, pues, lo que el discurso hace en o con el interlocu-

21 En este punto es importante subrayar que a lo largo del Gorgias Sócrates no ignora o niega la importancia de la persuasión, sino que la resalta al considerar la manera como esta se direcciona y qué pretende obtener: dinero, salud o poder, por ejemplo.

22 Como bien lo señala Dodds (1959, p. 30), el interés que podría despertar hoy día el Gorgias no depende de la dimensión formal o lógica de los argumentos dialécticos, que son raramente convincentes y, a veces, claramente falaces. 
tor y no meramente la consistencia formal. Aún más, se podría decir que cuando se trata de hacer que alguien se vuelva justo, tanto la claridad lógica como las posibilidades retóricas del lenguaje están orientadas a una conmoción afectiva del individuo en su enfrentamiento a lo público, por ejemplo, apelando a su orgullo y vergüenza. Como se muestra en el Gorgias, la inconsistencia lógica, expuesta públicamente, puede ser motivo de vergüenza, así como la victoria en el discurso puede ser motivo de orgullo. Se podría sostener, además, que la inconsistencia lógica tiene un valor ético o moral en la medida en que revela, cuando se trata de discursos sobre lo justo o lo injusto, que no se ha llegado a una opinión clara sobre temas de suma importancia política y, por tanto, que no se está en una posición conveniente para emitir juicios sobre ello ${ }^{23}$. En este sentido, se esperaría de alguien cuyas contradicciones en relación con lo justo y lo injusto se han puesto en evidencia que, si no cambia su opinión para adoptar opiniones que se revelen consistentes, al menos, suspenda el juicio al respecto.

En este orden de ideas, el trabajo del verdadero político que ejemplifica Sócrates, sería el de exponer la posición moral de los individuos, sus opiniones más íntimas sobre lo justo y lo injusto, apelando fundamentalmente al orgullo o la vergüenza, emociones que suponen, usualmente de manera implícita, la referencia esencial a una alteridad, por ejemplo, la opinión pública, la perspectiva de una comunidad o, en últimas, la mirada más profunda del propio yo. En este sentido, la dimensión performativa del lenguaje, que se identifica en este caso con su función política, sirve para revelar la vulnerabilidad del sujeto, su dependencia, inevitable, de la visión y opinión de un otro u otros. Cabe insistir en que lo importante políticamente no es solo la exposición de la dependencia respecto de los otros bajo la forma de la opinión pública. Esta exposición del individuo funciona, primordialmente, como el detonante de una conmoción emocional que incentiva una auténtica experiencia de la trascendencia, de exposición a la alteridad y de reconocimiento de lo que se impone como verdad, como evidencia, como necesidad, como ley. En otras palabras, el énfasis socrático en la dimensión performativa del lenguaje no solamente busca exponer el trasfondo de las opiniones políticas del individuo y su verdadera valoración moral de las mismas, sino promover el

$23 \mathrm{Al}$ respecto es importante anotar que en el Gorgias se sugiere que, en general, la ignorancia respecto de lo justo o lo injusto puede ser, en sí misma, algo vergonzoso. 
ejercicio dialéctico que, en tanto que lo obliga a ir más allá de sí mismo, sería condición necesaria para el conocimiento de los ideales del bien y la justicia.

Para explicar lo relativo al esfuerzo dialéctico que es necesario para el conocimiento de lo justo y lo bueno, es útil remitirse a lo que se dice en la Carta VII ${ }^{4}$ sobre el proceso para la aprehensión de las ideas. En esta carta se sugiere que el conocimiento de lo ideal no consiste en la mera adquisición de contenidos conceptuales sino un trabajo con el lenguaje, una especie de ritual laborioso con el que se posibilita un evento transformativo. En este sentido, vale la pena insistir que sobre ello lo importante no es la mera consistencia de un argumento o la precisión formal de una definición, sino la dimensión performativa del diálogo, el hecho de que el diálogo es una práctica y el terreno de una experiencia, que en la búsqueda benévola de precisiones terminológicas y argumentativas, en un intercambio de preguntas y respuestas, conduce al perfeccionamiento moral. Al respecto, el siguiente pasaje de la Carta VII es particularmente significativo:

Es necesario, en efecto, aprender ambas cosas a la vez, la verdad y lo falso del ser entero, a costa de mucho trabajo y mucho tiempo, como dije al principio. Y cuando después de muchos esfuerzos se han hecho poner en relación unos con otros cada uno de los distintos elementos, nombres y definiciones, percepciones de la vista y de los demás sentidos, cuando son sometidos a críticas benévolas, en las que no hay mala intención al hacer preguntas ni respuestas, surge de repente la intelección y comprensión de cada objeto con toda la intensidad de que es capaz la fuerza humana. (Carta VII 344b-c)

En este pasaje se sugiere que si bien, un adecuado discernimiento de vicios y virtudes supone tanto una profunda familiaridad con el lenguaje (nombres y definiciones) como una actitud benévola, surge del diálogo como una fulguración o irradiación ${ }^{25}$, de manera que la mente se expone a una especie de rapto, a una

24 Independiente de la posición que se asuma respecto de la autenticidad de esta carta, que es una cuestión controversial, se trata de un documento cuyos planteamientos iluminan el punto que estamos examinando.

25 La formulación en griego que corresponde a parte de la última línea citada reza: "' $\chi \chi \dot{\varepsilon} \lambda \alpha \mu \psi \varepsilon \phi p o ́ v \eta \sigma \iota \varsigma$

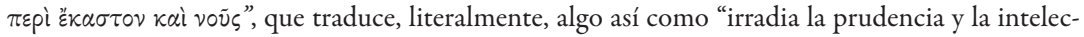
ción respecto de cada cosa". En este caso es importante tener en cuenta que el verbo $\dot{\varepsilon} \chi \dot{\varepsilon} \lambda \alpha \mu \psi \varepsilon$ es la conjugación en la tercera persona del singular, aoristo indicativo activo, del verbo $̇ \kappa \lambda \dot{\alpha} \mu \pi \omega$, que significa iluminar, radiar o fulgurar — en inglés to shine or beam forth, según se indica en la defini-

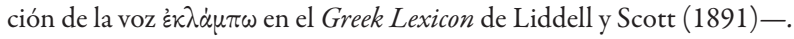


cierta experiencia de trascendencia, en tanto presiona los límites de nuestras capacidades intelectivas. Si relacionamos estos planteamientos con nuestro examen del Gorgias, podemos afirmar que el conocimiento relativo a lo justo, que es efectivamente una virtud o excelencia del alma, supone la singularidad de una experiencia y la aplicación de una ley no reducible al nivel de contenidos proposicionales y de inferencias deductivas: se trata siempre de un acontecimiento singular. En consonancia con esta aproximación, vale la pena recordar las advertencias de Platón respecto de la escritura, considerando que esta puede ser usada o asumida de manera equivocada, pues puede hacer pensar que el conocimiento es algo exterior al alma, algo que podría ser adquirido como información objetiva que se impone, como heteronomía, en ella.

El peligro sobre el cual nos advierte Platón en relación con la escritura se puede extender también a lo que significa nuestra relación con el lenguaje en general, cuando este no es usado con seriedad ${ }^{26}$ : esta falta de seriedad es evidente cuando las fórmulas lingüísticas se repiten sin que haya una apropiación real de sus significados e implicaciones, cuando estas no suponen una transformación existencial del individuo. Así pues, la referencia a un ideal o a un bien mayor no supone la subordinación del individuo en relación con un ideal universal sino que, por lo contrario, el bien mayor es el cuidado del individuo, el tratamiento curativo de su alma que supone una exhibición o exposición de su ser, de sus anhelos más genuinos. Entonces, el verdadero político trataría de llevar al individuo a una visión de lo bueno, no entendido como un contenido conceptual, sino como una transformación integral de la propia existencia. De esta manera, de acuerdo con lo que en parte argumenta Alexander Nehamas (1992, pp. 283-287), podemos decir que el arte político socrático no correspondería una visión ética positiva basada en conocimientos que se saben verdaderos, y que se pueden transmitir o enseñar como verdades, si bien —en parte contrariando lo dicho por Nehamas-Sócrates sí parece incentivar, enseñar y ejemplificar, una cierta actitud o disposición afectiva que, en últimas, podría entenderse como de respeto incondicional a la ley.

Finalmente, retomando la comparación entre el arte político y otras artes o disciplinas, y considerando el beneficio que este arte puede proporcionar a lapolis,

26 Según se sugiere en la Carta VII (344c - d), nadie seriamente preocupado por cuestiones relativas al vicio y la virtud podría tomarse en serio lo que está simplemente escrito. 
concluimos que la realización del bien mayor supondría una correlación armónica e incluso, diríamos, carnal, entre el individuo y la comunidad. Con esto se quiere significar, siguiendo lo planteado por David Sedley (2009), que el arte político socrático no contempla la violencia de hecho sobre el individuo, a lo sumo implica la violencia que se ejerce desde el lenguaje y la refutación. En efecto, como lo sugiere Sedley (2009), posiblemente el único castigo que el político puede operar es el de la refutación, castigo que causa sufrimiento en la medida en que el individuo se resiste a abandonar sus creencias, tendencia en cierta medida inevitable. Cabe aclarar que en este caso el castigo se presenta como un beneficio que solamente es comprensible como beneficio por fuera del círculo económico que entiende el castigo como un intercambio de maldad por maldad; antes bien, la justicia exigiría salir de este círculo para posibilitar, precisamente, la recepción de un bien que no se ha calculado y que no es en principio calculable, pues se trata de un bien que debe incluir idealmente a todo otro posible y por venir. Consecuentemente, el mensaje implícito en el mito del final del diálogo es que la búsqueda de la justicia, que supone la comprensión de la perspectiva de la otredad, exige siempre el abandono del mundo, de nuestro mundo: el ámbito del alma, de lo inmaterial, sería, así, el espacio de una apertura infinita, plasticidad infinita, libertad absoluta. De este modo, la injusticia, sería otro nombre para la negación, auto infligida, de la libertad, la negación de la apertura y la trascendencia.

Referencias

Dodds, E.R. (1959). Gorgias. A Revised Text with Introduction and Commentary. London: Oxford University Press.

Nehamas, A. (1992). What Did Socrates Teach and To Whom Did He Teach It? Review of Metaphysics, 46(2), pp. 279-306.

Liddell, H.G \& Scott, R. (1891). A Lexicon abridged from Liddell \& Scott's Greek-English Lexicon. New York: Harper \& Brothers.

Nussbaum, M. (1995). La fragilidad del bien. Fortuna y ética en la tragedia y la filosofía griega. Madrid: Visor.

Platón. (1988). Banquete. Diálogos III. (Trad. M. Martínez Hernández). Madrid: Gredos. 
Platón. (1993). Carta VII. Diálogos VII. Cartas. (Trad. J. Zaragoza \& P. Gómez Cardó). Madrid: Gredos.

Platón. (1993) Eutifrón. Diálogos I. (Trad. J. Calonge). Madrid: Gredos.

Platón. (1988). Fedón. Diálogos III. (Trad. C. García Gual). Madrid: Gredos.

Platón. (1967). Gorgias. (Trad. A. Cappelletti). Buenos Aires: Editorial Universitaria de Buenos Aires (EUDEBA).

Platón. (1988). República. Diálogos IV. (Trad. C. Eggers Lan). Madrid: Gredos.

Sedley, D. (2009). Myth, Punishment and Politics in the Gorgias. C. Partenie (Ed.). Plato's Myths (pp. 51-76). Cambridge, UK: Cambridge University Press.

Tarnopolsky, C.H. (2010). Prudes, Perverts and Tyrants. Plato's Gorgias and The Politics of Shame. Princeton: Princeton University Press. 